\title{
Modification of the circadian rhythm of onset of acute myocardial infarction by long-term antianginal treatment
}

Kent L Woods, Susan Fletcher, Carol Jagger

\begin{abstract}
Objective-To elucidate the mechanism of the circadian pattern of onset of acute myocardial infarction by examining the effects of prior antianginal treatment upon it.

Design-Retrospective analysis of clock time of the onset of acute myocardial infarction by linear modelling to define the circadian distribution of hourly onset rates and to examine the deviation of treated groups of patients from this distribution.
\end{abstract}

Setting-Coronary care unit in a general hospital taking unselected acute admissions from a district of 0.9 million people.

Patients-A series of 2231 patients with confirmed acute myocardial infarction.

Results-A major 24 h cycle and smaller $12 \mathrm{~h}$ and $6 \mathrm{~h}$ cycles were present in patients not taking antianginal medication. Onset rates varied twofold over the day, with maxima around 10.00 am and $10.00 \mathrm{pm}$. This pattern was unchanged in patients on prior treatment with regular nitrates, but in those who had been taking a $\beta$ blocker or a calcium antagonist the 24 $h$ cycle was absent.

Conclusions-These results are best explained by the shared property of $\beta$ blockers and calcium antagonists to reduce blood pressure and myocardial oxygen demand. The mid-morning peak of the onset of myocardial infarction is attributable to the physiological increase in sympathetic drive and cardiac work at that time. The data are not consistent with the triggering of the $24 \mathrm{~h}$ periodicity by fluctuations in coronary tone or haemostatic activity.

\section{(Br Heart J 1992;68:458-61)}

Acute myocardial infarction occurs as the end result of two sequential processes-coronary atherosclerosis and thrombotic occlusion. The risk factors for these two processes are unlikely to be the same though they cannot be distinguished by conventional epidemiological studies. A clear understanding of the trigger for acute thrombotic occlusion would provide scope for pharmacological primary prevention. A promising approach to the mechanism of the occlusive event is offered by the observation that the time of onset of acute myocardial infarction is not randomly distributed but shows a strong circadian rhythm with its major peak in the mid-morning and a minor peak in the late evening. ${ }^{12} \mathrm{~A}$ similar pattern has also been reported for sudden cardiac death. ${ }^{3}$ Possible explanations for this periodicity include physiological fluctuations in blood pressure, ${ }^{4}$ sympathoadrenal activity, ${ }^{5}$ platelet aggregability, ${ }^{6}$ or coronary tone. ${ }^{7}$

Evidence of modification of the circadian pattern by prior drug therapy would give insight into its mechanism. The use of observational data to identify drug effects requires particular care to control for confounding in the analysis. ${ }^{8}$ Muller et al found no circadian pattern of onset in patients entering the MILIS (Myocardial Infarction Limitation Intervention Study) trial with acute myocardial infarction who had been taking $\beta$ blocking drugs before admission but the statistical power was only $48 \%$ and concurrent use of other antianginal agents was not excluded. ${ }^{1}$ Willich et al also found no circadian periodicity of onset among patients with myocardial infarction entering the ISAM (intravenous streptokinase in acute myocardial infarction) trial who had been taking a $\beta$ blocker but they did observe a significant morning peak of onset among all trial entrants and in a subgroup who had been taking a calcium antagonist. ${ }^{9}$ For the whole UCSD (University of California at San Diego) Collaborative Database of 4796 patients there were two peak times of onset, the larger between 6.00 am and noon and the smaller between $6.00 \mathrm{pm}$ and midnight; in the subgroup who had been taking a $\beta$ blocker the two peaks were of equal size. ${ }^{2}$ The problem of coprescribing of antianginal drugs has not been addressed. In both the MILIS ${ }^{1}$ and the ISAM ${ }^{9}$ reports less than half the screened patients were included in the analysis of periodicity, so that selection bias could have arisen.

The present study was undertaken to examine the temporal distribution of the onset of acute myocardial infarction in a consecutive series of patients and to investigate the separate effects upon it of prior treatment with $\beta$ blockers, calcium antagonists, or nitrates. The important hypothesis to be tested is not whether patients in these treatment subgroups show a circadian pattern of onset of infarction, but whether the subgroups deviate significantly from the circadian pattern shown by patients not on prior chronic treatment with antianginal drugs. The first step in the present study was therefore to develop the best-fit 
mathematical model for the temporal distribution of onset of myocardial infarction in a large series of untreated patients. Treatment effects were then examined in groups of patients who had been receiving one or more antianginal drugs at the time of onset of acute myocardial infarction. The term "antianginal drug" refers here to $\beta$ blockers, nitrates (long-acting oral or transdermal preparations only), and calcium antagonists whether they had actually been prescribed for angina or for another indication. To avoid confounding of the drug effects by coprescription of more than one class of antianginal drug, their separate effects on circadian periodicity were identified by linear modelling.

\section{Patients and methods}

The patients included in the study were admitted with acute myocardial infarction to the coronary care unit of Leicester Royal Infirmary between January 1987 and September 1991. The diagnosis was based on the presence of at least two of the following: typical cardiac pain of at least 20 minutes' duration unrelieved by rest or buccal nitrates; evolving electrocardiograph changes of infarction; and a rise in the serum creatine kinase concentration to above twice the upper limit of the laboratory reference range. For each patient a detailed record of history, medication on admission, and other clinical data was completed on entry by medical staff and stored on computer without knowledge of the hypothesis to be tested. The clock time of onset of the symptoms of myocardial infarction was derived from the clock time of admission and the duration of symptoms as reported on admission. The clock time of onset of symptoms could not be estimated in $69(3.0 \%)$ of 2300 consecutive patients with confirmed acute myocardial infarction. The remaining 2231 patients formed the study group. Their mean age was 63 years. Figure 1 shows the subgroups by treatment. The calcium antagonists being used were examined by a random sampling of 100 sets of case-notes in this subgroup; $83 \%$ were taking a dihydropyridine (most commonly nifedipine), $15 \%$ diltiazem, and $2 \%$ verapamil. The number of patients $(8.6 \%)$ taking a non-steroidal anti-inflammatory drug regularly (including aspirin) was too small for separate analysis.

The data were formed into a contingency


table of the hour of day of onset by level of drug therapy with the cells of the table containing the number of individuals. To form the table the times of onset were rounded to the nearest hour, the boundaries being the surrounding half hours. Initially the levels of drug treatment were classified as no drug therapy, $\beta$ blockers (BB) only, calcium antagonists (CA) only, nitrates (NI) only, BB + CA, BB + NI, CA + $\mathrm{NI}$, and $\mathrm{BB}+\mathrm{CA}+\mathrm{NI}$. We fitted log linear models to this table using the statistical package GLIM $^{10}$ with a Poisson error structure. The clock time of onset was entered into the model in the form of paired sine and cosine transformations representing the harmonic components. The initial model (model 1) included harmonic terms up to the fourth order $(24,12$, 8 , and $6 \mathrm{~h}$ cycles) with levels of drug treatment. The model allowed a different baseline for each category of drug treatment and contained interaction terms between level of drug treatment and each sine and cosine term. Each level of drug treatment was therefore represented by a different wave form. From this model the effect of each of the harmonic components of the model was tested by dropping terms if the resulting change in deviance (with a $\chi^{2}$ distribution) was non-significant at the $1 \%$ level. The overall fit of the final model (model 2) formed by this step-down process was assessed by plots of the standardised residuals ${ }^{11}$ against the fitted values, time of onset, and level of treatment. From model 2 two further hypotheses were tested. The first was that any drug or combination had the same effect on the wave form of time of onset (the constant term representing the baseline being unaffected). The second hypothesis tested the equality of the constant term for any drug class or combination of drug classes.

\section{Results}

Figure 2 shows the raw data for the circadian distribution of onset of symptoms of myocardial infarction. Data for patients on no prior antianginal treatment $(n=1464)$ and those on any regular antianginal treatment $(n=767)$ are plotted separately.

Model 1, containing a constant term and four pairs of sine and cosine terms for each level of drug treatment, had a deviance of 11.0 on 120 degrees of freedom (df). Neither the fourth order harmonic $(p=0.75)$, third harmonic $(p$ $=0.51)$, nor second harmonic $(\mathrm{p}=0.81)$ were significantly different for the different levels of drug treatment. Checks on the contribution of the second, third, and fourth harmonic terms to the model showed that the third harmonic $(\mathrm{p}=$ 0.23 ) could be dropped but both the second ( $p$ $<0.0001)$ and the fourth harmonics ( $p=$ 0.004 ) added significantly to the model. Model 2 , therefore, had a different baseline level and first order harmonic for each class of drug together with a second and fourth order harmonic that were the same for all levels of drug treatment. The overall fit of this model (model 2) was good with a deviance of 146.4 on $164 \mathrm{df}$ and was supported by plots of the standardised residuals against the fitted values, time of onset, and level of drug treatment. 

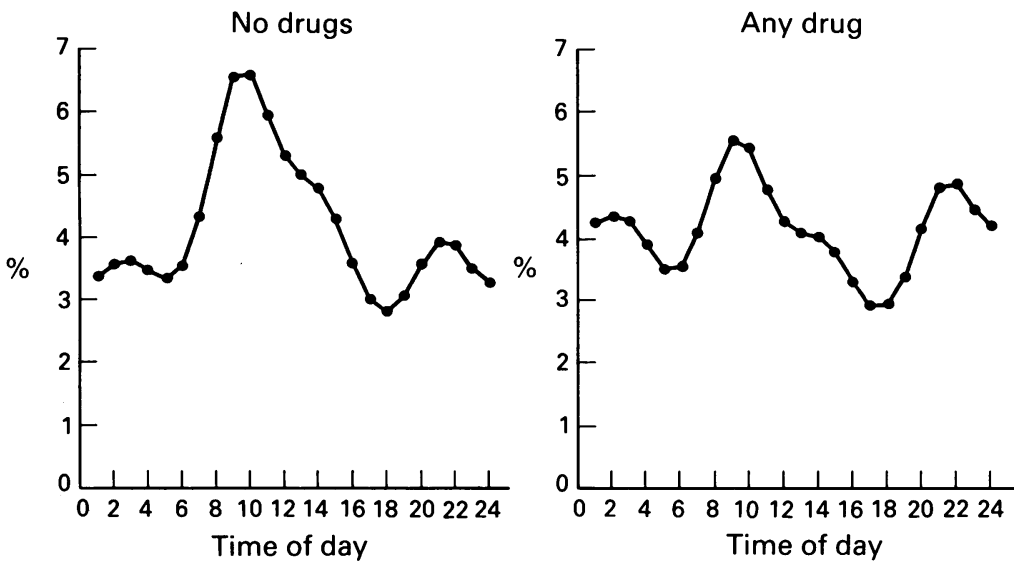

Figure 2 Observed distribution of time of onset of symptoms of myocardial infarction: $(A)$ for patients not taking antianginal drugs $(n=1464)$ and $(B)$ for patients on any antianginal drug treatment $(n=767)$.

Table Coefficients and standard errors of the terms in the final model (model 3)

\begin{tabular}{|c|c|}
\hline Term in the model & Coefficient ( $S E$ ) \\
\hline \multicolumn{2}{|l|}{ Constant +: } \\
\hline No drug & $4.08(0.03)$ \\
\hline BB & $2.03(0.07)$ \\
\hline CA & $1.69(0.09)$ \\
\hline NI & $1.96(0.08)$ \\
\hline $\mathbf{B B}+\mathbf{C A}$ & $0.83(0.13)$ \\
\hline $\mathrm{BB}+\mathrm{NI}$ & $1 \cdot 12(0 \cdot 12)$ \\
\hline $\mathrm{CA}+\mathrm{NI}$ & $1.40(0 \cdot 10)$ \\
\hline $\mathrm{BB}+\mathrm{CA}+\mathrm{NI}$ & $0.64(0.15)$ \\
\hline \multicolumn{2}{|l|}{ Cosine 1 st order harmonic: } \\
\hline No drug & $-0.24(0.04)$ \\
\hline Any drug & $0.01(0.05)$ \\
\hline \multicolumn{2}{|l|}{ Sine 1st order harmonic: } \\
\hline No drug & $0.11(0.03)$ \\
\hline Any drug & $0.09(0.05)$ \\
\hline Cosine 2nd order harmonic & $0.14(0.03)$ \\
\hline Sine 2 nd order harmonic & $-0.13(0.03)$ \\
\hline Cosine 4th order harmonic & $-0.11(0.03)$ \\
\hline Sine 4 th order harmonic & $0.01(0.03)$ \\
\hline
\end{tabular}
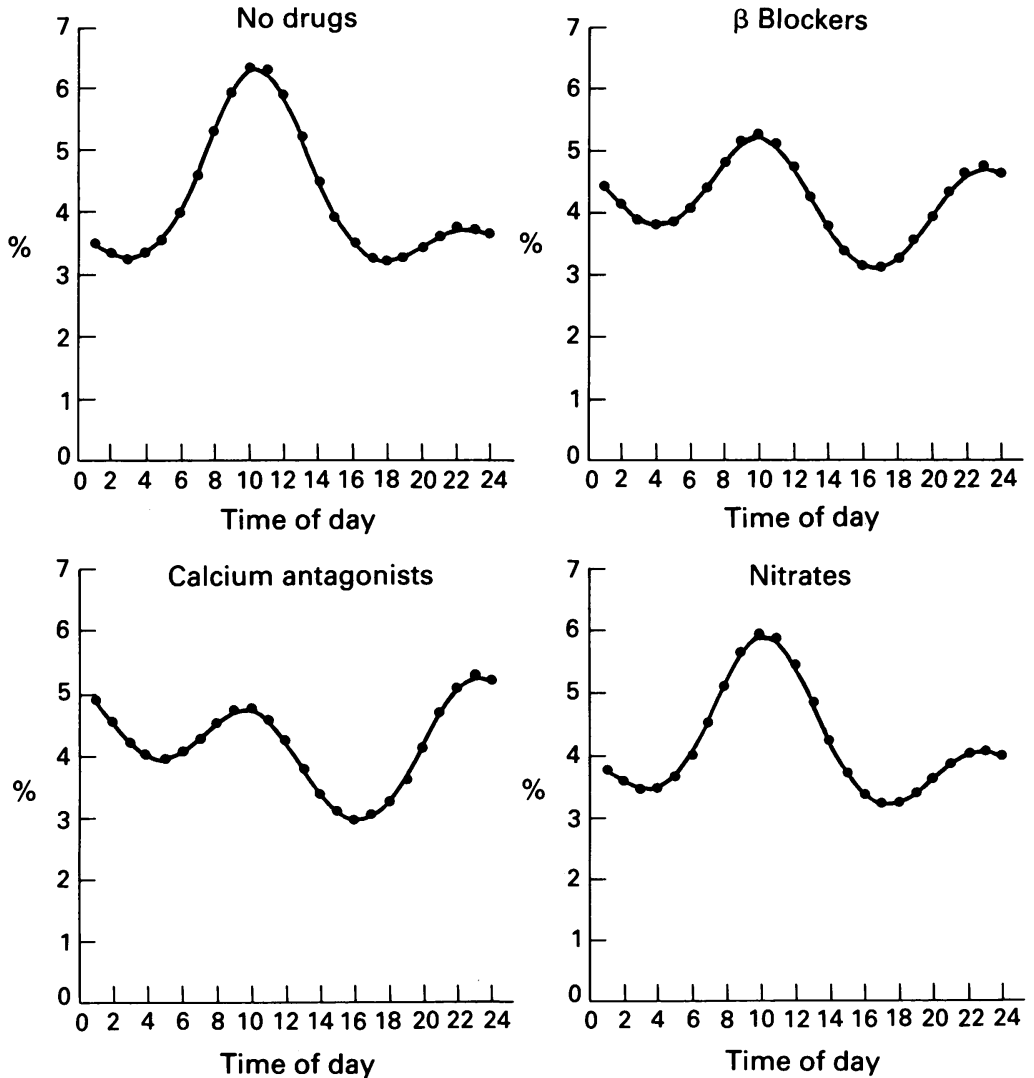

Figure 3 Fitted harmonic regressions (model 3) of the distribution of time of onset for patients $(A)$ not taking antianginal drugs, (B) taking blocker, $(C)$ taking a calcium antagonist, (D) taking nitrates regularly.
The next stage in the modelling process was to test the equality of the harmonic terms in those patients who had been receiving any regular antianginal treatment. The fit of this model (model 3) was not significantly worse than that of model $2(\mathrm{p}=0.68)$. The test of the second hypothesis, that the constant term was identical for all those on drug treatment, was rejected ( $\mathrm{p}<0.0001)$. A further model was fitted which allowed the constant term to be identical for combinations of two or more drugs only, but this again gave a significantly poorer fit to the data ( $p<0.0001)$. Plots of standardised residuals for model 3 showed no evidence of non-random scatter, and the overall deviance of 156.0 on $176 \mathrm{df}$ demonstrated that the model fitted the data well. The table shows the coefficients of the terms in Model 3 and their standard errors.

Figure 3 shows plots of the fitted distribution of onset throughout the $24 \mathrm{~h}$, using model 3 , for patients on no drug treatment and taking each class of anti-anginal drug. The clear circadian pattern seen in the patients on no drug treatment was unaffected by prior use of nitrates; in contrast, the dominant $24 \mathrm{~h}$ cycle with its peak around 10.00 am was abolished by $\beta$ blockers and calcium antagonists though in each case a residual $12 \mathrm{~h}$ cycle was clearly apparent.

\section{Discussion}

Timing the onset of acute myocardial infarction from the history has been shown to correlate very well with back-extrapolation from the rise in cardiac enzymes. ${ }^{19}$ Since all but $3 \%$ of patients with confirmed acute myocardial infarction were included in the analysis, the only factor that might have produced selection bias is death before the patient reached hospital. This is unlikely because out-ofhospital sudden cardiac deaths have been shown to have a similar harmonic distribution to that seen in survivors reaching hospital. ${ }^{3}$

The circadian pattern reported here has its maxima and minima within an hour of the "population rhythm" of the onset of acute myocardial infarction described by Muller et $a l .{ }^{1}$ The ISAM data9 ${ }^{9}$ generally accord with our results though spectral analysis of the ISAM data showed only one $(24 \mathrm{~h})$ cycle. In addition to the well recognised dominant $24 \mathrm{~h}$ periodicity, we saw the smaller 12 hour cycle noted in some studies of acute myocardial infarction as a late evening peak, ${ }^{12}$ and an additional minor $6 \mathrm{~h}$ cycle not previously described.

The main interest of the present study concerns the treatment subgroups. This type of analysis raises important issues of statistical inference that have usually not been adequately addressed. Firstly, the arbitrary division of the day into a small number of intervals to form a contingency table is insensitive and may introduce bias if data-derived. Use of hourly rates as dependent variables in a regression analysis has clear advantages. Secondly, statistical power will inevitably decrease as smaller subgroups within the whole sample are examined and the form of the null hypothesis will determine the consequences of a type II 
error. If the null hypothesis is that of a uniform distribution of cases over time, a type II error will lead to the incorrect conclusion that in contrast to the whole sample the subgroup lacks a circadian pattern. The appropriate null hypothesis is that the subgroup does not deviate from the best-fit circadian pattern previously identified in the whole sample: a type II error will then be conservative and will not lead to the same false inference. Thirdly, treatment subgroups have to be examined in such a way as to control for the common coprescription of major drug classes that was clearly seen in this data set. These analytical problems are best handled by the technique of linear modelling, specifying the Poisson error distribution appropriate to count data.

Although the mechanisms driving the $12 \mathrm{~h}$ and $6 \mathrm{~h}$ cycles are unknown, there are three plausible triggers to account for the $24 \mathrm{~h}$ cycle which causes the mid-morning peak and is suppressed by prior treatment with a $\beta$ blocker or calcium antagonist. There is evidence for suitably timed circadian variations in haemodynamic function, ${ }^{413}$ resulting in increased cardiac work; in coronary tone, ${ }^{14}$ which could precipitate plaque rupture; ${ }^{615} 16$ and in haemostatic function, predisposing to acute occlusion of an atheromatous coronary artery. ${ }^{6}$ Our data on the classes of antianginal drugs strongly support the first of these proposed mechanisms. $\beta$ Blockers and calcium antagonists by their antihypertensive and negatively inotropic effects will control the extra myocardial oxygen demand imposed by the known morning increase in sympathetic drive, blood pressure and heart rate. ${ }^{13}$ The observed abolition of $24 \mathrm{~h}$ periodicity of onset by $\beta$ blockers but not by regular nitrates indicates that circadian fluctuation in coronary tone (which has been described in patients with unstable angina ${ }^{17}$ ) does not cause the $24 \mathrm{~h}$ cycle of myocardial infarction, despite the evidence that coronary spasm can precipitate thrombotic occlusion by causing plaque rupture. ${ }^{75}$

Transient myocardial ischaemia detected by continuous ambulatory monitoring of patients with coronary artery disease shows both $24 \mathrm{~h}$ and $12 \mathrm{~h}$ periodicities with maxima around $10.00 \mathrm{am}$ and $9.00 \mathrm{pm} .{ }^{18}{ }^{19}$ The morning peak is suppressible both by atenolol ${ }^{18}$ and by nifedipine ${ }^{19}$; long-term nitrate treatment does not seem to have been investigated in this context. The close similarity of transient ischaemia and acute myocardial infarction in terms of their chronobiology and its modification by treatment suggests that the same triggering mechanism operates.

The present study design does not allow any direct measurement of the effect of prior drug treatment on the overall risk of myocardial infarction. A clearer understanding of the process whereby stable coronary artery disease progresses to acute occlusion may nevertheless guide treatment strategies for angina. These observational data give indirect evidence for a protective action of $\beta$ blockers and calcium antagonists that is not shared by long-acting nitrates. Definitive proof would require randomised trials of an unprecedented size and duration.

We thank the many medical and nursing staff of the coronary care unit who helped in data collection. SF is supported by a British Heart Foundation project grant.

1 Muller JE, Stone PH, Turi ZG, et al and the MILIS Study Group. Circadian variation in the frequency of onset of acute myocardial infarction. $N$ Engl J Med 1985;313:1315-22.

2 Gilpin EA, Hjalmarson A, Ross J. Subgroups of patients with atypical circadian patterns of sympton onset in acute myocardial infarction. Am J Cardiol 1990;66:7-11

3 Muller JE, Ludmer PL, Willich SN, et al. Circadian variation in the frequency of sudden cardiac death Circulation 1987;75:131-8.

4 Millar-Craig MW, Bishop C, Raftery EB. Circadian variation of blood-pressure. Lancet 1978;i:795-7.

5 Turton MB, Deegan T. Circadian variation of plasma catecholamine, cortisol and immunoreactive insulin concentrations in supine subjects. Clin Chim Acta 1974 55:389-97.

6 Tofler GH, Brezinski D, Schafer AI, et al. Concurrent morning increase in platelet aggregability and the risk of myocardial infarction and sudden cardiac death. $N$ Engl $J$ Med 1987;316:1514-8.

7 Maseri A, L'Abbate A, Baroldi G, et al. Coronary vasospasm as a possible cause of myocardial infarction. $N$ Engl J Med 1978;299:1271-7.

8 Miettinen OS. The need for randomisation in the study of intended effects. Stat Med 1983;2:267-71.

9 Willich SN, Linderer T, Wegscheider K, Leizorovicz A Alamercery I, Schroder R, and ISAM Study Group. Increased morning incidence of myocardial infarction in the ISAM study: absence with prior $\beta$ adrenergic blockade. Circulation 1989;80:853-8.

10 Baker RJ, Nelder JA. The GLIM system, Release 3. Oxford: Numerical Algorithms Group, 1978.

11 Aitkin M, Anderson D, Francis B, Hind J. Statistical modelling in GLIM. Oxford: Oxford University Press, 1989

12 Thompson DR, Sutton TW, Jowett NI, Pohl JEF. Circadian variation in the frequency of onset of chest pain in acute myocardial infarction. Br Heart J 1991;66:177-8.

13 Panza JA, Epstein SE, Quyyumi AA. Circadian variation in vascular tone and its relation to alpha-sympathetic vasoconstrictor activity. $N$ Engl J Med 1991;325:986-90.

14 Yasue H, Omote S, Takizawa A, Nagao M, Miwa K, Tanaka S. Circadian variation of exercise capacity in patients with Prinzmetal's variant angina: role of exercise-induced coronary arterial spasm. Circulation 1979;5:938-48.

15 Davies MJ, Thomas AC. Plaque fissuring: the cause of acute myocardial infarction, sudden ischaemic death, and crescendo angina. Br Heart $J$ 1985;53:363-73.

16 Richardson PD, Davies MJ, Born GVR. Influence of plaque configuration and stress distribution on fissuring of coronary atherosclerotic plaques. Lancet 1989;ii:941-4.

17 Waters DD, Miller DD, Bouchard A, Bosch X, Theroux P. Circadian variation in variant angina. Am J Cardiol 1984; 54:61-4.

18 Mulcahy D, Keegan J, Cunningham D, et al. Circadian variation of total ischaemic burden and its alteration with anti-anginal agents. Lancet 1988;ii:755-8.

19 Nesto RW, Phillips RT, Kett KG, McAuliffe LS, Roberts M, Hegarty P. Effect of nifedipine on total ischacmic activity and circadian distribution of myocardial ischaemic episodes in angina pectoris. $\mathrm{Am} \mathrm{J}$ Cardiol 1991;67:128-32. 